\title{
Retour sur les règnes, une immensité sidérante
}

Jean-Christophe Bailly

\section{OpenEdition}

\section{Journals}

Édition électronique

URL : http://journals.openedition.org/alter/626

DOI : $10.4000 /$ alter.626

ISSN : 2558-7927

\section{Éditeur :}

Association ALTER, Archives Husserl (CNRS-UMR 8547)

\section{Édition imprimée}

Date de publication : 31 décembre 2018

Pagination : 159-174

ISBN : 978-2-9550449-4-0

ISSN : 1249-8947

\section{Référence électronique}

Jean-Christophe Bailly, «Retour sur les règnes, une immensité sidérante », Alter [En ligne], 26 | 2018 mis en ligne le 31 décembre 2019, consulté le 25 mars 2020. URL : http://journals.openedition.org/ alter/626 ; DOI : https://doi.org/10.4000/alter.626 


\title{
RETOUR SUR LES RÈGNES, UNE IMMENSITÉ SIDÉRANTE
}

\author{
Jean-Christophe Bailly
}

Cette dimension d'immensité ${ }^{1}$ est la première qui me vient à l'esprit, dès lors qu'il est question d'aborder la nature. Nature est un mot qui avait presque disparu, de l'université en tout cas. À partir de lectures hâtives, de Foucault notamment, on en était arrivé à ne plus voir dans la nature que l'illusion voire le fantasme d'une originarité perdue. Or cela, je ne l'ai jamais pensé, je ne l'ai jamais cru, j'ai toujours pensé, au contraire, qu'avec $p h u s i s$ et bios, nous avions un infini de la composition (une immensité) qui, heureusement, échappait encore à la maîtrise des hommes - qui peut-être ne lui échappera pas toujours, mais en tout cas c'est encore comme ça. Assez récemment, alors que je fréquente cet auteur depuis cinquante ans maintenant, j'ai retrouvé dans Novalis deux formulations extrêmement frappantes et justes. Elles se trouvent dans Les Disciples à Sä̈s et non pas dans ce qu'on appelle l'Encyclopédie, que d'autres appellent le Brouillon général, que je relis sans cesse.

La première: «La somme de ce qui nous touche, on l'appelle la nature »- «was uns rührt», «rühren » cela veut dire « toucher », même « déranger», « émouvoir».

L'autre, qui est quelques lignes plus loin: «La nature est cette communauté merveilleuse (wunderbare Gemeinschaft) où nous introduit notre corps ». On est en 1801.

Je trouve ces formulations éblouissantes, et elles corrigent l'image assez caricaturale qui est donnée de la pensée occidentale comme si

\footnotetext{
${ }^{1}$ Ce texte est une transcription de la conférence de Jean-Christophe Bailly à l'École Normale Supérieure le 20 mars 2018 ; les éditeurs lui ont conservé son style oral, et, sauf mention contraire, les notes sont celles des éditeurs.
} 
elle était un tout, comme si elle n'était pas fracturée elle-même, disons, entre une attitude globalement anthropocentrique, dominante, avec la coupure radicale entre un sujet érigé le plus haut possible et des objets répartis en le plus de cases possibles, et une autre voie qui parie sur des continuités, des conductibilités, comme aimaient à le dire les romantiques allemands. On peut trouver des traces de cela dans la pensée mystique, à l'intérieur même du christianisme, lequel pourtant a joué un grand rôle dans l'institution de la créature comme supersujet. Dans certains aspects du franciscanisme, par exemple et, de façon beaucoup plus construite et élaborée, au sein de la pensée des romantiques allemands, et notamment de Novalis.

Donc à chaque fois qu'il y a des travaux d'ailleurs remarquables, comme ceux de Descola, nature, culture, etc., j'ai toujours envie de frapper à la porte du savant et de dire : oui monsieur mais, quand même, on n'a pas tous été comme vous dites que nous sommes. Et qu'est-ce que cela veut dire, la phrase de Novalis, justement? Elle est remarquable, surtout la seconde, parce qu'il y a cette idée de communauté, et parce que ce n'est pas une communauté vague, ce n'est pas une communauté imaginée ; c'est une communauté qui fonctionne à partir d'enclenchements, et ces enclenchements, $c^{\prime}$ est le corps qui les produit. Notre corps, cela veut dire nos sens, évidemment, notre respiration, notre existence même, en tant qu'êtres vivants, en tant qu'êtres sensibles. Donc la nature n'est pas quelque chose qui est détaché, qui est devant nous, c'est quelque chose dans quoi nous sommes et qui est également en nous - puisque, par exemple, respirer, simplement respirer, ou laisser-comme nous avons le bonheur de pouvoir le faire - le sang circuler en nous, c'est appartenir à quelque chose qui est de l'ordre de la nature, quelles que soient les possibilités de transfusions, de prothèses, etc. ; la base est toujours nature. Et, en tout cas, ces enclenchements, ce sont des relations. Au lieu d'être quelque chose devant quoi nous sommes, la nature est en quelque sorte continuée en nous, elle nous accompagne. Certes la pensée humaine et le langage en est le témoignage - va au-delà de tout cela, se détache de tout cela, mais c'est pour y revenir, et en tout cas, elle n'en est, pour moi en tout cas, jamais, jamais séparée. Et, dès lors que l'on dit cela on peut imaginer une quantité infinie de rapports. Il y a les rapports permanents, non pensés; il y a aussi les rapports que l'on noue avec tout ce qui surgit, qui peut être de l'ordre de l'infime. Et comme disait cet écrivain magnifique qu'est Robert Walser : «l'infime c'est déjà beaucoup ». C'est-à-dire qu'aussitôt que l'on se penche sur les choses, et ce peut être aussi bien le geste du savant (vers l'infiniment petit ou l'infiniment grand) que le geste de la seule pensivité, et à chaque fois 
- cela remonte à l'enfance -, on est stupéfait devant la quantité du sens qui surgit. Le sens non pas en tant qu'il est la signification que les hommes donnent aux choses, mais en tant qu'il est lui-même production permanente de signes, de signaux, d'intensités émanant du monde tel qu'il est autour de nous. Par exemple devant moi, dans cette salle, je ne vois a priori rien qui soit à proprement parler nature, et pourtant si, il y a quand même vos yeux, vos nez, votre peau, et si je me tourne vers la fenêtre, alors il y a le ciel ; les gens croient que la ville est totalement coupée de la nature. Mais j'aime toujours à dire - et c'est à peu près ce que je disais à Blois dès le premier jour aux étudiants paysagistes - le ciel commence à un centimètre de la terre, où que l'on soit. Voilà. Déjà pour moi cela pose un peu les choses, même si le ciel que l'on voit au bout de la rue n'est pas l'image la plus immédiate de la nature. Le réflexe est plutôt de se figurer l'équivalent d'un tableau de Caspar David Friedrich, plutôt qu'une vue urbaine. Il reste que la découpe des toits et même la façon dont le ciel est rendu visible par les tranchées urbaines que sont les rues, lui donne une présence singulière - et parfois c'est très fort, la nuit, ou dès lors qu'intervient le vent, sans même parler de la neige. La nature n'est pas absentée de la ville, pas encore absentée de la ville, et c'est d'ailleurs là un des grands bonheurs urbains.

Mais «le ciel commence à un centimètre de la terre », ce n'est pas seulement une formule, ce qui est désigné par là, c'est cette mince couche fabriquée par le vivant pendant des milliards d'années et qui le protège en retour, l'atmosphère, donc - que l'on peut légitimement considérer comme la part de ciel qui nous effleure et par laquelle commence déjà l'infini. Au-delà du tout, comme le disait Lucrèce, il n'y a rien qui le termine - je pense à cette image venue de la fin du MoyenÂge, où l'on voit un personnage agenouillé qui a passé la main au-delà du firmament, par-delà la voûte étoilée. Quelle que soit l'étendue des connaissances, et elle est aujourd'hui très grande, on reste avec cette sorte de puits sans fond inversé, l'immensité directement devant nous et sur nous. On n'y pense pas forcément en sortant de chez soi, mais dès qu'on a fermé la porte, on est immédiatement projeté dans l'univers, on est dehors. Et se retrouver dehors, être dehors, c'est en fait être dedans - en plein dedans, comme on dit. Dans quoi donc ? Mais dans la nature, en pleine nature, dans l'espace-temps qui nous héberge pour quelques temps. Il me semble qu'au fur et à mesure que se développent - et vous savez qu'elles le font de manière incroyablement exponentielle de nos jours - les connaissances et surtout les applications techniques de ces connaissances, on perd de vue ces dimensions fon- 
damentales de l'existence; or je crois que les rappeler est tout à fait nécessaire, à plein d'égards - y compris pour des raisons, disons, quasi politiques, et nous rejoindrions par là des problématiques liées à l'écologie, dans lesquelles je ne pense pas que nous aurons le temps d'entrer.

Donc je reviens sur cette notion d'infime: l'infime, c'est aussi, évidemment, le multiple, et l'autre leçon, qui est très facile à retenir que chacun connaît et que chacun oublie - c'est celle de l'infinie diversité du vivant et des contacts avec le vivant : ce qu'on appelle la biodiversité, à quoi je tiens à ajouter encore - et c'est pour cela que je tiens à parler de ce qu'on appelait autrefois les règnes - la richesse du monde minéral. Diversité qui se déploie non seulement d'une espèce à une autre mais qui, à l'intérieur de chaque espèce, se divise en singularités, à l'infini. Ce qui veut dire que toute existence est singulière et que la « communauté merveilleuse » de Novalis doit être repensée à l'aune de cette diversité, de cette multiplicité des diversités singulières. C'est une communauté presque impensable, mais qui pourtant existe et fonctionne. Et on voit d'ailleurs aujourd'hui, que cette manière de voir, qui étend le commun au-delà de la limite humaine, se répand. Je pense par exemple à ce livre qui s'intitule Comment pensent les forêts ${ }^{2}$, dans lequel la réflexion sur les possibilités d'une communauté extraordinairement étendue, qui n'inclurait plus seulement les hommes, les groupes humains, mais tout ce qui les entoure, se fonde sur le suivi de groupes humains réels qui ne sont ni des peuples non contactés ni des populations intégrées dans la banlieue générique. Mais notre civilisation, la civilisation technique s'est entièrement coupée de ces modes de pensée et de réflexion évoqués dans le livre d'Eduardo Kohn.

Pour illustrer ce que j'ai à dire, il y aurait cette fameuse phrase d'un chef Inuit citée, d'ailleurs, par Descola, qui disait à un explorateur norvégien : " mais vous comprenez, le plus grand péril - c'est comme ça que c'est dit - le plus grand péril de l'existence, c'est qu'il n'y a rien d'autre à manger que des esprits ». Comme vous le savez, dans le grand Nord, on ne peut pas cultiver la terre, on ne peut pas faire pousser de plantes, donc on est condamné, si l'on veut vivre, à manger de la viande, donc à tuer des êtres, tuer des esprits. Et ce qui est très frappant $c^{\prime}$ est que cette pensée - c'est une pensée - qui se comprend aisément en milieu arctique, on la voit finalement imprégner la plupart des sociétés de chasseurs-cueilleurs: le fait de tuer n'est pas automatique, et pas même celui de cueillir. À chaque fois on risque, on

2 E. Kohn, Comment pensent les forêts. Vers une anthropologie au-delà de l'humain, Le Kremlin-Bicêtre, Éditions Zones Sensibles, 2017. 
prend un risque, et il faut garantir, se garantir. C'est la fameuse logique de la dette, dans laquelle entre le sacrifice. Toutes ces précautions sont évidemment totalement oubliées ou négligées aujourd'hui, et c'est quelque chose, évidemment, de très dommageable.

Si l'on veut s'approcher un peu plus des contenus sensibles et matériels, ce qui permet de le faire, c'est justement la question des règnes, la question donc de tout ce qui existe, la question de ce avec quoi et dans quoi nous sommes. Ici, la passion du rangement, qui cherche à répondre à la profusion, est fondamentale. Et je prendrai l'exemple de Linné. Linné, si on ne le lit pas, si on ne va pas y voir de plus près, peut passer pour une caricature de la pensée qui classe, qui range, et qui répartit. Mais ce qui est extraordinaire, quand on le lit (son Voyage en Laponie est un livre merveilleux - au sens du merveilleux de Novalis) -, c'est qu'on est en compagnie de quelqu'un qui explore le monde sous nos yeux et que littéralement tout, tout, requiert ; tout requiert son attention, tout l'étonne. Linné, et c'est merveilleux aussi, a un usage spontané du latin - il parle latin. Et par exemple ça donne : ce matin, je me suis réveillé, il faisait tant de degrés, et j'ai trouvé - excusez-moi, j'invente un nom - j'ai trouvé Scapula infans devant ma tente, etc., et puis il part sur la description de cette plante, et puis il y a des morceaux en latin ; deux pages après il donne la recette $\mathrm{du}$ fromage de renne, il trouve un collier, il dessine une cabane, la forme d'un outil, le plan anthropologique s'enroule autour de son plan principal, botanique, mais à peu près tout, tout le requiert, le saisit. Donc ce Voyage, quand on le lit, c'est un énorme bric-à-brac, si l'on peut dire, de données ; et ce bric-à-brac de données, ensuite, il a eu l'idée, le désir de l'organiser - et à mon avis ce n'est pas encore fait, il y aurait à écrire une histoire du réflexe toponymique, qui est peut-être ce qu'il y a de plus intéressant chez l'homme. Par exemple, si on pense à des objets vraiment lointains comme la lune : à peine a-t-on découvert le moyen de la regarder mieux, avec les lunettes, qu'on y voit des choses, des taches, des plis, des cicatrices, et à peine les a-t-on vus qu'on leur donne des noms. C'est tout à fait étonnant. Et d'ailleurs les Grecs définissaient l'inconnu comme a-metriton - ce qui n'a pas encore été arpenté - un rivage ametriton c'est aussi, bien sûr, un rivage innommé. Plutôt que de penser le langage comme le signe et la preuve d'une supériorité de l'homme sur toutes les autres créatures - j'ai pensé que le langage était la forme humaine du frayage, la manière humaine de frayer dans le monde. Trouver des noms et les articuler par le langage. Mais justement, il y a bien d'autres frayages, et c'est ce à quoi je veux en venir, car ces autres frayages sont, pour le nôtre, des sources et des ressources. 
Alors vous le savez, on disait qu'il y avait trois règnes - ce n'est plus guère utilisé, mais enfin, les minéraux restent plutôt minéraux, les végétaux restent plutôt végétaux, et les animaux restent plutôt animaux ! Mais j'en ajoute un quatrième finalement - qui est celui qui se dirige vers ce qu'on appelle l'anthropocène - c'est-à-dire qu'au sein des animaux, il y en a quand même un qui est très spécial, c'est l'homme, et je crois que nous devons le mettre à part. Ce qui donne quatre règnes, et surtout quatre formes de temporalité qui leur correspondent. Il y a donc quatre formes de règnes : le règne minéral, le règne végétal, le règne animal, et le règne, disons, humain. Bien entendu il y a quelque chose de chronologique dans la succession de ces règnes ; mais il est à noter qu'aucun n'a effacé l'autre et que nous sommes pris aujourd'hui dans un devenir qui tisse ensemble ces règnes et les formes de vie et d'existence qu'ils ont suscité - tout cela va de pair et fonctionne. Et là, on est obligé pour penser la nature pour penser à la nature - de faire un insert sur la question, il me semble, du temps, de la forme du temps, et de voir que cette temporalitélà fait fonctionner des ensembles qui sont très différents; elle donne une idée du temps que je m'étais amusé à caractériser en réfléchissant à des choses que j'avais, un peu par hasard, découvertes en regardant du côté de la câblerie, autrement dit des techniques de production des câbles. Et j'avais été très intéressé par la structure des câbles - n'étant ni mathématicien ni physicien, c'était juste une curiosité, pensive disons, et si je savais que tout câble était une tresse, j'ai appris que pour qu'un câble tienne, il fallait qu'il y ait, au centre de la tresse, un vide. Ce vide au centre de la tresse s'appelle l'âme. Et, immédiatement, j'ai pensé au temps comme à un câble, comme à un tel câble. (Je sais que des théories physiques compliquent ou rendent caduque toute image linéaire du temps, mais pour ce que j'ai à dire peu importe que le câble fasse un vortex ou n'importe quoi d'autre, il reste quand même linéaire au niveau de notre expérience empirique.) Et je le vois ainsi : composé d'une infinité de petites fibres, qui seraient les fils passagers de l'expérience et les fils continus des conditions d'expérience offertes par les différents règnes - avec au centre quelque chose qui serait comme un vide, qui serait l'âme du temps, qui serait ce qui tient que le temps soit, ce qui tient qu'il tienne. À partir de là on pourrait entrer dans des considérations ontologiques extrêmement complexes, vertigineuses et passionnantes, mais je vais essayer de nous replacer au niveau où interviennent les temporalités des différents règnes.

La grande différence qu'il y a entre le règne minéral et les autres, c'est qu'il est assigné à résider dans phusis, alors que les autres, ceux des animaux, des plantes et les hommes, ce serait plutôt phusis + bios. 
Mais cette frontière, comme on peut le vérifier, n'est pas nette, puisque natura naturans - le fait que la nature est toujours à elle-même son propre laboratoire, et qu'elle est toujours en train d'évoluer et de changer - cela concerne aussi, bien entendu, le règne minéral. Simplement, et c'est là la temporalité du minéral, les changements y sont beaucoup plus lents. Et avec les pierres, et ce qui relève des pierres, on est dans quelque chose d'autre que Roger Caillois, qui était passionné par elles, a caractérisé comme « un mystère plus lent, plus vaste et plus grave que le destin d'une espèce passagère ». C'est là le premier rapport que l'on a au monde minéral, même si ce monde minéral se rappelle aussi à nous par des secousses extrêmement violentes. Puisque le minéral qui est, disons, la couche la plus ancienne - puisque c'est même ce qui nous porte, donc la planète elle-même - le minéral, c'est le sidéral. Et là on sort complètement de l'allégorie ou de la métaphore - comme je vous disais tout à l'heure : le ciel commence à un centimètre de la terre - c'est que la Terre elle-même est quelque chose de post-stellaire, si on peut le dire ainsi, et que nous habitons un fragment de ciel, et que ce qui se souvient d'avoir été un fragment de ciel, c'est le monde minéral. Le minéral et le sidéral, c'est strictement la même chose. Et quand je vous disais que cela se rappelle à nous, évidemment que vous le savez bien, il y a d'une part le tellurique, le volcanique, et tout ce qui ressortit aujourd'hui à ce que Wegener a révélé avec la dérive des continents, etc. Donc nous habitons ce devenir, mais ce devenir minéral est, heureusement pour nous d'une certaine manière, d'une quasi - on ne peut pas dire mieux que quasi d'une quasi stabilité. Pour illustrer cela, la chose qui me viendrait à l'esprit, c'est un monument humain, ce sont les alignements de Carnac ou les cromlechs comme on en voit de formidables dans les pays celtiques y compris d'ailleurs les plus beaux au Portugal. Ce sont ces pierres posées directement sous le ciel et dont vous savez très bien qu'elles avaient une fonction d'observatoire, c'est-à-dire que ces hommes très anciens, dont on sait extrêmement peu de choses, ces hommes du néolithique, eh bien ils avaient, eux, entièrement conscience de cette continuité terre-ciel, de cette continuité minéral-sidéral que j'évoquais. Et pour faire un petit insert sur l'exposition qui s'est tenue il y a peu au musée Zadkine, rue d'Assas - j'ai écrit dans le catalogue, je n'étais pas du tout responsable de cette exposition mais j'étais très heureux d'y être associé d'une certaine façon - elle s'appelait " Être pierre », et le titre « Être pierre » indique qu'il s'agissait via cette exposition d'une réflexion sur l'existence minérale. On peut rappeler ici - c'est toujours utile, en fait - la fameuse formulation de Heidegger sur les animaux qui ont peu de monde, les hommes qui sont constructeurs de monde 
et les pierres qui, elles, les pauvres, n'ont pas de monde du tout - ne serait-ce que pour signaler tout ce que cette exclusion des pierres de toute possibilité d'accès au sensible comporte de problématique - ce que j'ai essayé de dire dans le texte :

\begin{abstract}
Il n'est sans doute pas question d'aller prêter aux pierres des intentions ou une capacité d'expérience - une sensibilité. Mais pourtant l'on ressent que le jugement qui les exclut de toute vie, en les installant pour toujours dans une forme inerte de la présence, comporte quelque chose d'impensé, d'expéditif, d'injuste; elles vivent entièrement en elles-mêmes [ça c'est la citation d'un philosophe], oui, mais cette vie qu'elles ont, elles l'ont non seulement parmi d'autres formes de vie totalement différentes, mais en la portant malgré tout comme une vie, c'est-à-dire comme un devenir, si lent soit-il. Ce qui est en jeu ici, c'est l'ancrage ontologique si singulier des pierres, c'est cette façon qu'elles ont de passer dans l'être en y restant plus longtemps, beaucoup plus longtemps que la plupart des autres étants. [...] Le sobre enfoncement en soi d'une force de condensation, l'équilibre atteint entre une évidente malléabilité et une dureté sans concession, l'efficacité d'une installation têtue dans le nonagir, ce sont toutes ces qualités qui font des pierres, émissaires principaux $d u$ règne minéral des comparses au fond très étran-ges ${ }^{3}$.
\end{abstract}

Voilà : «Des comparses »-et je tiens absolument à maintenir cette expression.

Vous le voyez, tout cela est assez expéditif, rapide, trop rapide, mais on n'en a quand même pas encore fini avec le monde minéral. Il convient en effet d'ajouter quelque chose sur les cristaux, qui offrent, disons, l'image de la plus grande pérennité possible, de la plus grande dureté, en même temps qu'ils incarnent une rectitude géométrique naturelle qui devrait couper court aux spéculations sur le caractère nécessairement artificiel de la ligne droite. Mais le cristal, c'est aussi ce qui s'oppose, selon le couple classique, à la fumée, c'est-à-dire au plus évanescent, et il est important de souligner cet écart formel énorme, qui nous permet d'imaginer, entre les deux extrêmes, une gamme infinie.

Passons maintenant au règne végétal, aux plantes. À cette autre temporalité qu'ils incarnent. Avec eux on entre dans le vivant, et la définition la plus simple en est qu'est vivant tout ce qui finit par mourir. Est vivant ce qui meurt. Ce à quoi on peut aussi ajouter qu'est vivant ce qui se nourrit et se reproduit ; les plantes comme les animaux vivent, meurent, se nourrissent et se reproduisent, ce que les roches ne font pas, même si elles ont une sorte de vie. Mais la différence fondamentale qui divise le vivant entre les plantes et les animaux, c'est

\footnotetext{
${ }^{3}$ J.-Ch. Bailly, « Souveraineté des pierres » in Être pierre, catalogue de l'exposition qui s'est tenue au
} Musée Zadkine du 29 septembre 2017 au 11 février 2018, Musée Zadkine - Paris Musées, p. 18 sq. 
ce que l'on a appelé l'autotrophie pour les plantes et l'hétérotrophie pour les animaux. Ce que cela veut dire, c'est que les plantes qui vivent et se nourrissent $\mathrm{n}^{\prime}$ ont pas besoin de se déplacer pour le faire, alors que ce qui définit le règne animal, c'est la nécessité de se mouvoir, la nécessité d'errer en quête de sa nourriture. Les plantes, comme vous le savez, se déploient à la fois vers le haut et vers le bas; avec les mécanismes de la photosynthèse elles transforment ce qui leur vient du ciel (lequel commence à un centimètre de la terre !) - tandis qu'elles puisent dans le sol l'eau et les nutriments dont elles ont besoin, ce qui fait que chaque plante, du brin d'herbe au séquoia, fonctionne comme un point de suture entre la terre et le ciel. Et ce qui est extraordinaire, c'est que cette immobilité captive (leur enracinement), est compensée par une recherche formelle éperdue ; cette recherche formelle éperdue vers le haut, c'est ce qu'on appelle l'arborescence, mais vers le bas, il y a aussi une recherche formelle constante, via les racines, dont le déploiement est dans l'ensemble moins connu, moins étudié que celui des branchages. L'un comme l'autre réservent encore bien des surprises ; ce sont d'ailleurs des objets mathématiques presque incalculables, impensables, avec des difficultés énormes, et on pourrait poser comme examen mathématique : calculez la surface d'une pivoine, calculez la surface d'un chêne - c'est infaisable, infaisable! Le mouvement des plantes est en soi extraordinaire, puisqu'il est lié à la pesanteur, au sol, et en même temps antigravitationnel puisqu'il monte vers le haut, et peut-être avez-vous vu l'exposition de ce merveilleux artiste qu'est Giuseppe Penone $^{4}$ dans les jardins de Versailles. Ce sont des arbres qu'il utilise ou qu'il coule dans le bronze et, dans les embranchements il pose des rochers; donc ceux-ci au lieu de reposer au sol comme la gravitation le suppose restent suspendus entre les branches, ce qui donne une sculpture extraordinaire, qui contracte en un seul objet plastique la force gravitationnelle attirant les pierres vers le bas et la pulsion ascendante tirant les plantes vers le haut.

Quand je vous dis que les plantes compensent par une recherche formelle éperdue, cela concerne toute l'arborescence, et l'arbre, littéralement, palpe le monde autour de lui, aussi longtemps qu'il vit. Les plantes sont des êtres vivants qui travaillent, qui sont sans cesse au

\footnotetext{
${ }^{4}$ Sur les expositions de ses œuvres, voir, par exemple, http://mediation.centrepompidou.fr/ education/ressources/ENS-penone/penone.html http://www.chateauversailles.fr/resources/pdf/fr/presse/dp_penone.pdf

et, sur l'exposition à la Villa Médicis, https://www.franceculture.fr/emissions/tout-arrive/ exposition-giuseppe-penone-la-villa-medicis-gilles-barbier-et-xavier-veilhan (consultés le 13 avril 2018).
} 
travail - sauf l'hiver; et dès que le printemps arrive, ça recommence et c'est véritablement une palpation, une exploration : comment vais-je faire pour prendre le plus de lumière? L'existence et la forme des branches, la forme des rameaux et la forme des feuilles, c'est toujours le résultat de ce travail ; voilà comment faire pour attraper le plus de lumière, et évidemment cela change selon les conditions, les latitudes, les espèces, etc., mais c'est absolument merveilleux de voir ce travail prodigieux effectué par les plantes quelles qu'elles soient, même les très petites. Les hommes peuvent intervenir en les mettant dans des serres pour augmenter la luminosité ou modifier la température, mais l'opération reste intégralement celle du végétal avec la photosynthèse. En même temps cette immobilité n'est pas absolue, c'est le fameux chêne et le roseau, et le vent le montre ; le vent fait que, tout en étant immobiles, les plantes et les arbres réagissent au vent. Si elles n'étaient pas structurées pour réagir au vent, si elles n'étaient pas ces structures souples, balancées, eh bien elles casseraient et ne pourraient pas vivre. $E t$, là, on entre dans quelque chose qui illustre d'une certaine manière cette temporalité des végétaux qui est leur souplesse, leur manière d'habiter le temps, en acceptant le plus possible, en se comportant en quelque sorte, par rapport au vent ou aux forces de la nature, comme des sortes de hamacs, si on peut dire. Du côté des racines, on trouverait évidemment quantité de choses, à commencer par la collaboration permanente que le monde racinaire entretient avec le monde microbien; par exemple toutes les racines sont en interaction avec un microbiote qui les entoure un peu comme un gant de filaments. Ceuxci, certes, parasitent la plante, mais en même temps la protègent, il y a une véritable collaboration entre la plante et les microbes, tout le tissu formateur des champignons. On a découvert récemment que les plantes, par les racines et parfois aussi par les feuilles se communiquent des informations, relatives à un stress hydrique ou à une attaque par un animal, un insecte ou une girafe (les acacias par exemple). La communauté merveilleuse, c'est aussi cela.

On en vient donc aux animaux, c'est-à-dire à cette autre temporalité fondée sur le fait d'être coupé du milieu nourricier, d'avoir à se déplacer constamment - que l'on vive dans l'air, dans l'eau ou sur terre. Ce que cela crée, c'est l'urgence, une temporalité de recherche qui introduit des vitesses, qui rompt avec le mode fondamentalement patient des végétaux. Tout le monde connait cette célèbre mimique de Gilles Deleuze parlant de l'animal dans son Abécédaire : l'animal c'est l'être aux aguets. Oui, il a raison: l'être aux aguets, c'est la forme première de toutes les formes de vie animale, même les plus infimes. 
Par exemple, dans un livre très récent sur le sens de la vue Jean Deutsch, un spécialiste de cette science que l'on appelle evo devo (evolutionary developmental biology, c'est-à-dire la biologie évolutive du développement), explique que les méduses voient grâce à un système de captation de la lumière ${ }^{5}$. Il parle aussi, entre autres choses, d'une expérience qu'il a vécue dans un laboratoire de Roscoff lorsque seul, alors que la nuit tombait, il s'est senti regardé, observé : en fait il l'était, mais par tout un groupe de coquilles Saint-Jacques placé derrière lui ! Ce qui fait qu'on apprend qu'elles voient, qu'elles disposent d'un système de perception visuelle. La coquille Saint-Jacques, un des rares animaux que je me sentais non coupable de dévorer à pleines dents! Je ferme cette parenthèse, mais je vais en venir aux yeux proprement dits, non plus ceux de la méduse ou de la coquille Saint-Jacques, mais ceux des mammifères, des oiseaux, des reptiles, des poissons. Là, il y a une véritable différence non pas de la forme de l'être, mais de la forme de la relation; elle change du tout au tout dès lors qu'il y a regard. La communauté merveilleuse devient peut-être plus palpable même si elle transite par une forme de peur, elle devient plus palpable dès lors qu'il y a regard, échange visuel. Il faut faire l'équation œil-sang. Avec la plupart des animaux, nous partageons d'avoir du sang - plutôt que de la sève - et d'avoir des yeux plutôt que des stomates. Ce bien commun perceptif avec les animaux m'a énormément intrigué et séduit, et j'ai essayé dans la mesure du possible de l'étudier, de le penser en termes génériques. Disons pour aller vite que l'hétérotrophie a produit la nécessité pour ces corps d'avoir des moyens de se déplacer et d'identifier des nutriments, les proies si vous voulez; et la forme de chaque animal, que ce soit un poisson, un oiseau, un reptile, un mammifère, etc., la forme de son corps est le résultat d'un très très long travail de relation, de dialogue, de discussion - c'est un mot employé par Canguilhem, je crois - avec le milieu, le biome, dans lequel l'animal en question se retrouve. La nageoire par exemple, la serre, ce sont de formidables outils d'ingénierie qui correspondent à la nécessité pour l'animal de se nourrir de cette manière-là et pas d'une autre ; après c'est d'une complexité énorme, parce qu'il y a non seulement des formes spécifiques, mais aussi tout ce qui régit les interspécificités, puisque la communauté merveilleuse n'est pas une communauté idyllique mais qu'elle est aussi structurée par des chaines alimentaires et le fait que les animaux éventuellement s'entre-dévorent et le fait qu'ils utilisent constamment des nutriments venant du monde végétal.

${ }^{5}$ J. Deutsch, La méduse qui fait de l'œil et autres merveilles de l'évolution, Paris, Seuil, "Science ouverte $», 2017$. 
Donc le travail de la communauté merveilleuse, c'est un travail qui n'est pas une pure symphonie heureuse; alors, au niveau de la temporalité, du fait de la motricité et des outils de la mobilité, du fait des sens comme moyens mis au point par les espèces pour garantir les moyens de vivre, il y a un dédoublement qui vient à partir du rapport sensible; et dans Le versant animal, j'emploie l'expression de "nappe phréatique du sensible » : c'est comme s'il y avait une nappe phréatique du sensible commune à tous les animaux, et à laquelle ils auraient tous accès, mais chacun avec des moyens différents, comme si on buvait à cette nappe avec une paille différente pour la tortue, pour le caméléon, pour l'oiseau ou pour l'insecte, mais cette nappe phréatique du sensible est en fait partagée par tous.

Il y a des formes visibles de cette communauté silencieuse que l'on a avec les animaux, qui sont particulièrement émouvantes pour moi, par exemple quand on voit un animal, en général c'est presque toujours un mammifère, quand on voit un animal qui dort avec son corps qui se soulève, qui respire, on peut au moins dire qu'il est en train de faire la même chose que nous et de partager avec nous cet air qu'il respire. Mais on peut aller plus loin et justement par le regard on accède à un niveau étrange de communication; c'est une communication vide d'une certaine manière. Il n'y a pas là - et ça j'y tiens une dimension d'empathie ou fusionnelle ; on reste chacun du côté d'un seuil, mais la consistance de ce seuil est extraordinaire, et je peux raconter cette anecdote que je n'avais pas vécue, je crois, au moment où j'écrivais Le versant animal, car sinon je l'y aurais sans doute inséréé Celle de la rencontre avec une chauve-souris dans ma cave.

Au-delà du fait que je m'étais intéressé aux chauve-souris, entre autres, à leur système d'écholocation, qui vient en complément de leur vue qui est excellente, je suis entré dans la cave et la chauve-souris s'est mise à tournoyer comme elles le font, et je me suis immobilisé et elle aussi ; et elle s'est accrochée, avec leur extraordinaire aptitude à être à elles-mêmes leur propre hamac, et puis elle était peut-être à un mètre, un mètre vingt, accrochée, et puis on s'est regardés, il n'y a pas d'autre mot. C'est très petit une chauve-souris : vingt, trente grammes, $c^{\prime}$ est comme une hirondelle ; on voit son cœur battre. J'ai eu le plaisir, ne bougeant pas, de voir ce battement se ralentir, se calmer; on se regardait. Qu'est-ce qu'elle voyait? Moi, oui, comme moi je la voyais. Mais cela reste comme un seuil vide ; exactement comme le chien ou le chat à la maison, par moments, vous l'avez remarqué, même si vous le connaissez, il vous regarde comme s'il ne vous avait jamais vu. C'est

${ }^{6}$ J.-Ch. Bailly, Le versant animal, Paris, Bayard, 2007. 
insondable ce regard, et là il y aurait beaucoup à dire, sur la pensée, la pensivité, le silence. On peut étudier cela en se demandant seulement comment les animaux se servent de la vue (qui est chez beaucoup d'entre eux bien plus performante que la nôtre), et comment elle est toujours adaptée à ce dont ils ont besoin; mais en même temps il est évident qu'il y a un décalage entre l'amplitude de ce qu'ils voient et la nécessité dans laquelle ils sont de s'en servir pour la quête de leur nourriture ; et j'avais eu le plaisir en travaillant sur les animaux pour la radio de rencontrer un grand spécialiste des éléphants, Pierre Pfeffer ${ }^{7}$; $j^{\prime}$ avais été le voir au Jardin des plantes et il m'avait dit, vous savez la plupart de mes collègues étudient les animaux en cage, c'est un peu comme si un martien venait sur terre et faisait un rapport uniquement en allant à la prison de la Santé, et puis, pour les films animaliers, on les filme toujours - et c'est vrai, malheureusement -, le lion, le léopard, on les filme toujours en train de chasser, c'est exactement comme si on faisait un reportage sur les hommes uniquement quand ils vont chez le boucher, ce qui dans la journée n'occupe pas si longtemps... en dehors de ceux qui ne mangent plus de viande; le rapport à la viande, c'est exactement pareil pour les animaux, du fait de ces vitesses incroyables liées à tout un monde furtif. Il est clair, et j'ai eu le plaisir de le vivre sur place dans la savane en faisant un travail sur les animaux avec le peintre Gilles Aillaud, qu'il y a un autre usage du sens de la vue et cette différence ou ce surplus, nous pouvons l'étendre à tout le sensible. Par exemple à ce sens que nous avons mais à peine, par exemple le toucher ; on s'en sert mais c'est intéressant de voir comment chez quelqu'un qui devient aveugle le toucher se développe, etc. Mais, sans être aveugle, un lynx, par exemple, qui est le plus silencieux de tous les félins avec des coussinets extraordinaires avec toutes ses vibrilles, il est clair qu'il habite en même temps que nous dans le monde mais qu'il est dans un autre monde, un monde où le sens du toucher est exacerbé. Ces usages exacerbés des sens et cette vigilance aux aguets, c'est un peu ça ce que j'appelle la pensivité, et je n'attribue pas aux animaux forcément la pensée. On pourrait aller jusqu'à trouver une pensivité, disons, passive du côté des végétaux et peut-être même des pierres.

Comment comprendre l'idée de cette communauté merveilleuse ${ }^{8}$ ? Si le monde est l'entremêlement, l'entrelacement de tous les Umwelten, comme dit Uexküll, ces derniers fonctionnent à la fois séparément et

\footnotetext{
7 P. Pfeffer, Vie et mort d'un géant : l'éléphant d'Afrique, Paris, Flammarion, 1989.

${ }^{8}$ Cet alinéa précise le sens de la notion de communauté en réponse à une question posée à l'issue de la conférence.
} 
ensemble. Et l'idée de communauté désigne aussi bien celle de la famille, du village, du pays jusqu'à l'humanité, sans qu'il y ait de raison pour laquelle nous devrions nous arrêter à elle et nous empêcher de l'étendre au-delà de l'humain. La communauté plus large, c'est la conscience d'avoir d'abord en commun quelque chose, et le fait que la communauté ne doive pas se fermer est au fondement de toute pensée politique émancipatrice : les plantes, les animaux, les pierres, tout ça on l'embarque là-dedans; et si on pense très sérieusement à cet en commun, cela remet en cause énormément de pratiques humaines, que ce soit celles des industries qui n'ont aucun égard pour rien, ou celle d'un chasseur qui tire dans le tas. Les expériences du seuil - c'est Benjamin qui a parlé de cela -, pour moi sont fondamentales, ce sont justement des expériences de l'altérité, mais, comme on dit en banlieue, " respect»; c'est comme ça qu'on peut avancer, en s'arrêtant, en marquant le pas devant ce qui fabrique un seuil, et c'est très important qu'il y ait ces seuils. Malheureusement, on vit dans un monde où ceux qui sont au pouvoir empêchent les expériences de seuil ; ce sont les expériences de l'altérité à partir desquelles il peut y avoir mise en commun ; on met en commun l'altérité, on ne met pas en commun la similitude. Je me souviens de cette phrase de Novalis (oui, encore) citée par André Breton: "Éloignement infini du monde des fleurs » ${ }^{9}$; ça c'est l'attitude fondamentale d'une pensée comme celle que j'essaye de développer et qui serait peut-être la pensée romantique allemande, le contraire absolu de Lamartine, cette espèce d'empathie molle qui veut tout faire fusionner. Il faut partir de cette étrangeté, regarder vraiment ce qu'elle est, comment elle fonctionne, comment elle vit. Si peu longtemps, d'ailleurs: c'est incroyable cette brièveté, on parlait des temporalités... J'ai parlé de la temporalité végétale en évoquant ses longues durées, mais la temporalité végétale ce sont aussi des rythmes, des vitesses, puisqu'il y a une temporalité arbre, mais aussi celle des plantes annuelles, celle de la reproduction et de l'éclosion, et la temporalité florale avec parfois des durées extrêmement brèves..., que les papillons connaissent bien puisque c'est la leur, puisqu'ils vivent dans cette temporalité-là.

\footnotetext{
9 Voir Novalis, Fragmente II, Kapitel 2, " Unendliche Ferne der Blumenwelt. Schauspielertalente », 2235 - Sammlung aus dem Projekt Gutenberg-DE (Dresden, Jess Verlag, 1929), et Les disciples à Saïs et les fragments de Novalis, traduit de l'allemand et précédé d'une introduction de M. Maeterlinck, Bruxelles, Paul Lacomblez Éditeur, 1914, p. 197, qui rend cette phrase ainsi : «Le monde des fleurs est un infini lointain »https://archive.org/stream/lesdisciplessa00nova/lesdisciplessa00nova_djvu.txt (consulté le 13 avril 2018).
} 
Je n'ai pas eu le temps de parler des échanges; et c'est à partir de là qu'il devient intéressant de se demander : «mais qu'est-ce que j' aurais en commun avec cette tulipe? » J'aurais pu prendre "rose» par exemple, je suis obsédé par ça, parce que la région du Kenya où nous avons habité quand nous avons fait avec Gilles Aillaud et Frank Bordas ce travail sur les animaux, elle est aujourd'hui métamorphosée. Il y a là-bas un lac qui s'appelle le lac Nakuru, or entre le temps où nous étions là-bas (il y a trente ans) et maintenant, le niveau de ce lac a baissé de moitié. Pourquoi ? Parce que l'eau est systématiquement pompée par les cultivateurs de roses. Ces roses que vous trouvez ici dans les boutiques $A u$ nom de la rose ou qui sont vendues dans des restaurants (par des gens qui n'y sont pour rien), ce sont des roses qui sont en train de détruire l'écosystème entier d'une région. Si vous regardez la carte, vous ne voyez que des cercles cultivés et plus rien autour, ce sont des cercles d'arrosage et tout ce qui poussait a été éliminé, avec des techniques redoutables, une pratique qui est répandue aussi dans d'autres régions, pour d'autres productions, au Brésil... Et vous avez ces roses qui arrivent ici et qui d'ailleurs, comme vous savez, n'ont pas d'odeur, c'est là tout un symbole. Là on voit la capacité de nuisance humaine. Dans ces boutiques-là, du genre Au nom de la rose, la rose, en fait, n'est jamais regardée, elle n'existe d'ailleurs qu'en bouquet et le bouquet c'est aussi et déjà un objet qui engage un certain rapport. Il y a un livre magnifique de Jack Goody qui s'appelle La culture des fleurs et qui porte justement sur les rapports que les civilisations ont élaborés avec les fleurs ${ }^{10}$.

Pour terminer, je vous lirai la phrase de Plotin que j'avais mis en exergue du Versant animal; elle va aussi dans le sens de cette noncontinuité de la pensée occidentale et prouve que les Grecs, ce n'est pas seulement la version académique qu'on tire de Platon, c'est évidemment plus complexe : «Toute vie est une pensée, mais une pensée plus ou moins obscure comme la vie elle-même ». Et, juste en conclusion, je vous ai amené un texte de ce livre qui s'appelle L'oiseau Nyiro qui est un recueil des portraits d'animaux que j'ai faits avec Gilles Aillaud ; lui les dessinait, moi je les décrivais par l'écriture. Ce fut fait sur place, nous avons passé de longues semaines en Afrique Noire au Kenya ${ }^{11}$ pour être avec eux et les voir de plus près. C'est le premier texte qui s'appelle « Paysages », et ce sera ma conclusion :

\footnotetext{
10 J. Goody, La culture des fleurs (1993), Paris, Seuil, 1994.

${ }^{11}$ J.-Ch. Bailly, L'Oiseau Nyiro, Genève, La Dogana, 1991, rééd. 2012.
} 
Des traces, des pistes, des ondulations, des ponctuations, des groupes, des troupeaux, des zones sans rien, des zones remplies, du silence et, parfois, des bruits. Des trous d'eau, du bois mort, des couverts, des pierres parsemées de petites crêtes, des broussailles, une rivière, un feu et les bruits de pas qu'on entend dans le feu, des froissements, des frémissements, sur les robes, le soleil et la lune verticaux, les ombres ramassées, des masses qui se rassemblent, se séparent, des éclats de couleurs, d'oiseaux, des stries, des balancements, des paresses allongées dans l'herbe, des petits pas, des cavalcades, de la méfiance, du guet, de l'indolence, des distances, une tactique permanente du maintien des distances, des lois, c'est là, c'est donc là, le pays des animaux où ils sont chez eux et entre eux, sans parole et sans fable-immense vacance qui décide de la présence, silence total qui contient tous les bruits, visibilité entière de toutes les cachettes, scénario continu d'affleurements et de disparitions ; ce qu' on voit, on le devine, ce qu' on devine, on ne le voit plus, on est à l'intérieur, entièrement à l'intérieur et c'est ce que dehors veut dire, dedans en plein dedans, sans rémission, comme au sein d'une mémoire si vive qu'elle n'aurait pas de souvenirs, aucun stock ; de la présence uniquement, qui diffuse et qui infuse dans la lumière, ou dans la nuit. 